\title{
Characterization of the Schizosaccharomyces pombe Spt5-Spt4 complex
}

\author{
BEATE SCHWER, ${ }^{1}$ SUSANNE SCHNEIDER, ${ }^{1}$ YI PEI, $^{2}$ ANNA ARONOVA, ${ }^{1}$ and STEWART SHUMAN ${ }^{2}$ \\ ${ }^{1}$ Department of Microbiology and Immunology, Weill Cornell Medical College, New York, New York 10065, USA \\ ${ }^{2}$ Molecular Biology Program, Sloan-Kettering Institute, New York, New York 10065, USA
}

\begin{abstract}
The Spt5-Spt4 complex regulates early transcription elongation by RNA polymerase II and has an imputed role in pre-mRNA processing via its physical association with mRNA capping enzymes. Here we characterize the Schizosaccharomyces pombe core Spt5-Spt4 complex as a heterodimer and map a trypsin-resistant Spt4-binding domain within the Spt5 subunit. A genetic analysis of Spt4 in $S$. pombe revealed it to be inessential for growth at $25^{\circ} \mathrm{C}-30^{\circ} \mathrm{C}$ but critical at $37^{\circ} \mathrm{C}$. These results echo the

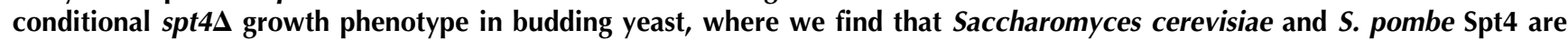
functionally interchangeable. Complementation of $S$. cerevisiae spt4s and a two-hybrid assay for Spt4-Spt5 interaction provided a readout of the effects of 33 missense and truncation mutations on $S$. pombe Spt4 function in vivo, which were interpreted in light of the recent crystal structure of $S$. cerevisiae Spt4 fused to a fragment of Spt5. Our results highlight the importance of the Spt4 $\mathrm{Zn}^{2+}$-binding residues-Cys12, Cys15, Cys29, and Asp32-and of Ser57, a conserved constituent of the Spt4-Spt5 interface. The 990-amino acid $S$. pombe Spt5 protein has an exceptionally regular carboxyl-terminal domain (CTD) composed of 18 nonapeptide repeats. We find that as few as three nonamer repeats sufficed for $S$. pombe growth, but only when Spt4 was present. Synthetic lethality of the $s p t 5^{1-835} \operatorname{spt} 4 \Delta$ double mutant at $34^{\circ} \mathrm{C}$ suggests that interaction of Spt4 with the central domain of Spt5 overlaps functionally with the Spt5 CTD.
\end{abstract}

Keywords: elongation checkpoint; mRNA capping; transcription elongation

\section{INTRODUCTION}

Eukaryal mRNA processing is coordinated physically and temporally with transcription elongation. The earliest processing step is mRNA capping, which can occur as soon as the $5^{\prime}$ triphosphate terminus of the nascent RNA extrudes from the elongating RNA polymerase (Chiu et al. 2002). The capping enzymes are directed to nascent mRNAs by binding to the phosphorylated carboxyl-terminal domain (CTD) of the largest subunit of RNA polymerase II (Cho et al. 1997; McCracken et al. 1997; Yue et al. 1997; Ho and Shuman 1999; Pei et al. 2001; Fabrega et al. 2003). Capping enzymes also bind to the Pol II elongation factor Spt5 (Wen and Shatkin 1999; Pei and Shuman 2002), which, in conjunction with its binding partner Spt4, elicits an

Reprint requests to: Beate Schwer, Department of Microbiology and Immunology, Weill Cornell Medical College, New York, NY 10065, USA; e-mail: bschwer@med.cornell.edu; fax: (212) 746-8587; or Stewart Shuman, Molecular Biology Program, Sloan-Kettering Institute, New York, NY 10065, USA; e-mail: s-shuman@ski.mskcc.org; fax: (212) 772-8410.

Article published online ahead of print. Article and publication date are at http://www.rnajournal.org/cgi/doi/10.1261/rna.1572709. elongation arrest at promoter-proximal sites (Wada et al. 1998a). The Spt5/Spt4-induced elongation arrest in metazoans is alleviated by the C $\mathrm{dk} 9$ protein kinase, a subunit of positive transcription elongation factor $\mathrm{b}(\mathrm{P}-\mathrm{TEFb})$ (Wada et al. 1998b). In the fission yeast Schizosaccharomyces pombe, the essential Cdk9 kinase exists as a stoichiometric complex in vivo with its cyclin partner (Pch1) and the methyltransferase component of the $S$. pombe capping apparatus (Pei and Shuman 2003; Pei et al. 2006; Guiguen et al. 2007). S. pombe Cdk9 also interacts with the RNA triphosphatase component of the capping apparatus (Pei et al. 2003). These and other observations underlie the proposal of a transcription elongation checkpoint that ensures a temporal window for capping of nascent mRNAs (Pei et al. 2003; Mandal et al. 2004; Sims et al. 2004). Evidence is emerging that positive and negative regulation of transcription can occur at the step of capping enzyme recruitment (Chiu et al. 2002; Gao and Gross 2008).

The Spt5-Spt4 complex is the lynchpin of the putative checkpoint. There is ample genetic and biochemical evidence from budding yeast, zebrafish, roundworm, fruitfly, and mammalian systems that Spt5 and Spt4 exert both 
negative and positive effects on transcription elongation (Hartzog et al. 1998; Andrulis et al. 2000; Guo et al. 2000; Renner et al. 2001; Shim et al. 2002; Wu et al. 2003; Jennings et al. 2004). Spt5 is a large polypeptide $(\sim 1000$ 1200 amino acids) composed of multiple domain modules, including an acidic N-terminal domain, central NusG-like NGN (NusG N-terminal homology), and KOW domains (Hartzog et al. 1998; Wade et al. 1998a; Ponting 2002), and a CTD targeted for threonine phosphorylation (Pei and Shuman 2003; Yamada et al. 2006). Spt5 is essential for cell growth in budding and fission yeast (Swanson et al. 1991; Pei and Shuman 2002). Saccharomyces cerevisiae Spt5 participates in a vast network of genetic and physical interactions with the Pol II elongation complex and mRNA processing systems (Lindstrom et al. 2003; see also Saccharomyces Genome Database, www.yeastgenome.org). By contrast, Spt4 is a small polypeptide (100-120 amino acids) and is nonessential for cell growth in budding yeast (Malone et al. 1993). Yeast spt4s cells display pleiotropic phenotypes that are probably linked to defects in transcription elongation and RNA processing (Rondón et al. 2003; Lindstrom et al. 2003; Mason and Struhl 2005; Qui et al. 2006; Schneider et al. 2006). Many genetic interactions with spt4s have been observed in budding yeast (Saccharomyces Genome Database, www.yeastgenome.org).

Here we conducted a physical and genetic characterization of the $S$. pombe Spt5-Spt4 complex that addressed the following issues: (1) the native size and subunit stoichiometry of a recombinant core Spt5-Spt4 complex; (2) structure probing of the complex by limited proteolysis; (3) whether S. pombe Spt4 can function in lieu of S. cerevisiae Spt4 in vivo; (4) identification of functionally important amino acids of Spt 4 via alanine scanning and conservative substitutions; (5) the effect of genetic ablation of Spt4 in S. pombe; and (6) synthetic enhancement of $S$. pombe Spt5 mutations by ablation of Spt4.

\section{RESULTS AND DISCUSSION}

\section{Recombinant S. pombe Spt5-Spt4 complex}

The human Spt5-Spt4 complex was purified initially based on its elongation-arrest activity. Wada et al. (1998a) estimated by gel filtration a native size of $>300 \mathrm{kDa}$ and suggested that the Spt5-Spt4 complex is a multimer of the $165-\mathrm{kDa}$ Spt5 protein. Deletion analysis delineated a central Spt4-binding domain on human Spt5 from amino acids 176-313 (Yamaguchi et al. 1999; Ivanov et al. 2000), but the methods employed (GST pull-downs, co-immunoprecipitations) did not reveal the stoichiometry or size of the complex.

Our aim here was to assess the quaternary structure of the core $S$. pombe Spt5-Spt4 complex formed by recombinant subunits. Based on our initial two-hybrid data showing that both the N-terminal 164-amino acid segment and the C-terminal 190-amino acid nonamer array of $S$. pombe Spt5 are dispensable for binding to Spt4 (Pei and Shuman 2002), we sought to physically characterize the complex of Spt4 with Spt5-(165-800). This was accomplished by coexpressing His-tagged Spt4 with untagged Spt5-(165-800) in bacteria and then purifying His-Spt4 by Ni-agarose chromatography (Fig. 1). The affinity-purified material (Fig. 1B, lane Ni) consisted of the 14-kDa His-Spt4 plus the untagged $70-\mathrm{kDa} \operatorname{Spt5}-(165-800)$ polypeptide in near 1:1 stoichiometry. This result proves that Spt4 and Spt5 associate in the absence of other fungal proteins. The $\mathrm{Ni}$-agarose preparation was sedimented in a glycerol gradient with internal markers catalase (a 248-kDa tetramer of a $62-\mathrm{kDa}$ subunit), bovine serum albumin (BSA) (a 68$\mathrm{kDa}$ monomer), and vaccinia topoisomerase (vtopo) (a 36$\mathrm{kDa}$ monomer). Analysis of the gradient fractions by SDSPAGE showed that Spt5 sedimented as a single discrete component coincident with the BSA peak and clearly heavier than the vtopo peak (Fig. 1A). There was no detectable heavier component of Spt5 between BSA and catalase, arguing against Spt5-(165-800) dimerizing. The fact that the majority of the Spt 4 cosedimented with Spt5 suggests that the complex is probably a $1: 1$ heterodimer, although we cannot exclude the possibility that two copies of the Spt4 polypeptide are associated with Spt5-(165-800). The residual "free" Spt4 sedimented as a lighter shoulder. We subjected the peak glycerol fraction containing Spt5Spt4 and the markers to another round of Ni-affinity purification, which resulted in efficient recovery of HisSpt4 and untagged Spt5, but not the markers (Fig. 1B, cf. lanes $\mathrm{G}$ and $\mathrm{G}+\mathrm{Ni}$ ), thereby attesting to the stability of the reconstituted Spt5-(165-800)-Spt4 complex.

We probed the structure of the isolated Spt5-(165-800)Spt4 complex by limited proteolysis with trypsin (Fig. 1C). We observed a discrete set of tryptic fragments that persisted at trypsin levels in excess of the amount sufficient to cleave all the input Spt5-(165-800). The N-terminal sequences of the input Spt5 and the tryptic fragments were determined and are indicated in Figure $1 \mathrm{C}$ at right (note the Spt5 N-terminus includes 3 amino acids upstream of Asp165 introduced during cloning into the bacterial expression vector). The protease-accessible sites in Spt5 were thereby mapped to the Arg217-Ser218, Lys381Asn382, and Lys466-Gly467 dipeptides. These sites could demarcate surface-exposed loops or interdomain linkers within the Spt5 tertiary structure. The changes in the spectrum of the proteolytic fragments as a function of trypsin concentration provide insight to the structure of the Spt5Spt4 complex. For example, at the lowest trypsin concentration, we see a $\sim 55-\mathrm{kDa}$ fragment initiating at Ser218, consistent with initial incision at a single major tryptic site at Arg217-Ser218. The predicted short N-terminal peptide (amino acids 165-217) product of initial cleavage was not seen in Figure 1C, because it would migrate well ahead of the intact Spt4 protein and be compressed at the 
A

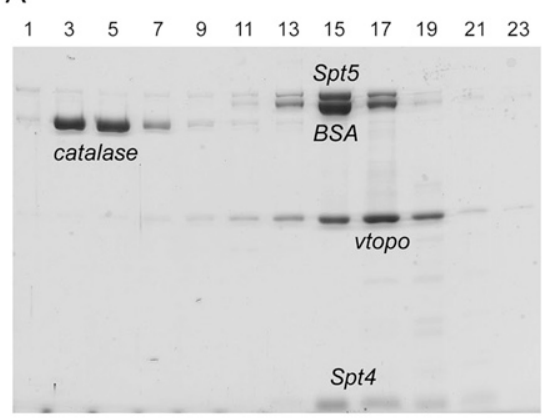

B

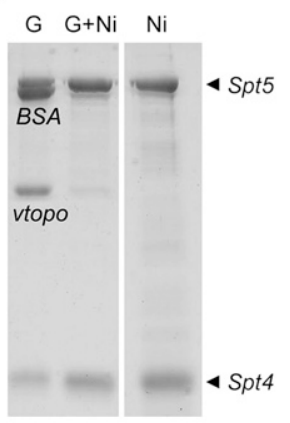

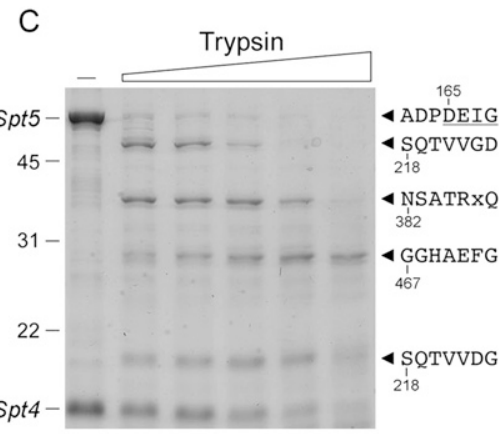

D

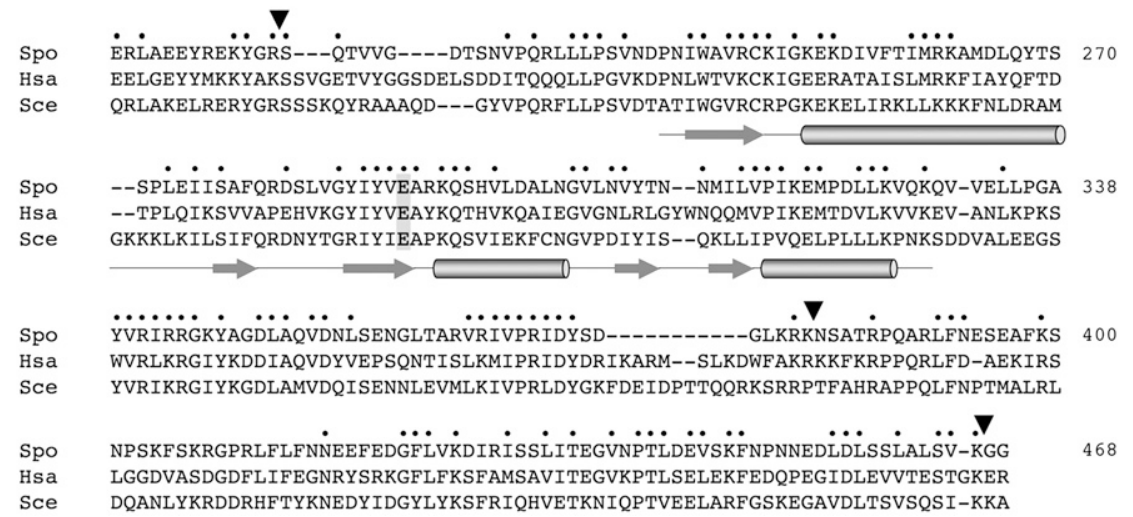

FIGURE 1. Characterization of a recombinant fission yeast Spt5-Spt4 complex. (A) Zonal velocity sedimentation of the HisSpt4/Spt5-(165-800) complex with internal standards was performed as described in Materials and Methods. Aliquots of the odd-numbered glycerol gradient fractions were analyzed by SDS-PAGE; the Coomassie blue-stained gel is shown. (B) Comparison by SDS-PAGE of the polypeptide compositions of the Niaffinity purified HisSpt4/Spt5-(165-800) complex that was applied to the glycerol gradient (Ni), glycerol gradient fraction 15 containing a mixture of HisSpt4/Spt5-(165-800), BSA, and vtopo (G), and the imidazole eluate obtained by subjecting an aliquot of glycerol gradient fraction 15 to a second round of Ni-affinity purification $(\mathrm{G}+\mathrm{Ni})$. The Coomassie blue-stained gel is shown. $(C)$ Limited proteolysis and automated Edman sequencing were performed as described (Lehman et al. 1999). Affinity-purified HisSpt4/Spt5-(165-800) was treated with 3.1, 6.2, 12.5, 25 or $50 \mathrm{ng}$ of trypsin. Trypsin was omitted from the control mixture in lane -. The digests were resolved by SDS-PAGE. Polypeptides were transferred electrophoretically to a polyvinylidene difluoride membrane and visualized by staining the membrane with Coomassie blue dye. Membrane slices containing intact Spt5-(165-800) and individual proteolytic products denoted by arrowheads were excised and sequenced. The N-terminal peptide sequences are indicated on the right. (D) The amino acid sequence of S. pombe (Spo) Spt5 from residues 205-468 is aligned to the sequences of the homologous human (Hsa) and S. cerevisiae (Sce) Spt5 proteins. Positions of side-chain identity or similarity in all three proteins are indicated by - The secondary structure elements of the NGN domain of S. cerevisiae Spt5 are displayed below the alignment (with $\beta$-strands depicted as arrows and $\alpha$-helices as cylinders). The mapped sites of tryptic cleavage of Spt5 in the recombinant $S$. pombe Spt 4 -Spt 4 complex are denoted by $\boldsymbol{\nabla}$.

electrophoretic front. The apparent size of the largest tryptic fragment was less than the value of $64 \mathrm{kDa}$ calculated for Spt5-(218-800), which suggests that trypsin also cleaved near the $\mathrm{C}$ terminus to yield one or more small fragments that are not visualized in the experiment. Also present at low trypsin concentrations were an $\sim 37$-kDa polypeptide initiating at Asn382 and an $\sim 18-\mathrm{kDa}$ species that retained the N-terminal Ser218 of the initial $55-\mathrm{kDa}$ tryptic fragment. The size of the smaller species agrees with the calculated size of the Spt5-(218-381) fragment. The $37-\mathrm{kDa}$ and $18-\mathrm{kDa}$ fragments persisted as trypsin concentration increased and all of the initial $55-\mathrm{kDa}$ species decayed (Fig. 1C). In turn, the $37-\mathrm{kDa}$ species was converted at the highest trypsin concentrations to an $\sim 29$-kDa derivative initiating at Gly467.

The small number of discrete tryptic cleavage sites in Spt5-(165-800), which has 81 lysine and arginine residues, suggests that the protein is well folded in the complex with Spt4. The locations of the three trypsin-sensitive sites in $S$. pombe Spt5 are denoted by arrowheads above the aligned amino acid sequences of the $S$. pombe, human, and $S$. cerevisiae Spt5 proteins (Fig. 1D). The Spt 4 binding domain of $S$. pombe Spt5 had previously been localized by twohybrid assays to the segment from amino acids 165-400 that includes two of the tryptic sites, Arg217 and Lys381 (Pei and Shuman 2002). Coexpression in bacteria of HisSpt4 and Spt5-(218-800) resulted in a stable complex of the two polypeptides after Ni-affinity chromatography (data not shown), suggesting that the entire polypeptide segment preceding the tryptic site at Arg217 is not strictly necessary for interaction with Spt4. The segment between the two most sensitive tryptic sites (Arg217 and Lys381) in S. pombe Spt5 corresponds to amino acids 147-329 in human Spt5, which embraces tightly the mapped binding domain for human Spt4 
(Yamaguchi et al. 1999; Ivanov et al. 2000). The apparent resistance of this segment of the S. pombe Spt5-Spt 4 complex to trypsin cleavage suggests that it comprises a folded domain.

This inference is underscored by the recently published crystal structure (Guo et al. 2008) of a chimeric protein composed of S. cerevisiae Spt4 fused to the homologous segment of $S$. cerevisiae Spt5 (amino acids 285-375). The structure revealed that the yeast Spt5 fragment adopts a compact tertiary structure composed of a central antiparallel $\beta$ sheet flanked by three $\alpha$ helices. The Spt5 secondary structure elements are depicted below the aligned sequences in Figure 1D.

\section{Use of budding yeast for structure-function analysis of $S$. pombe Spt4}

Whereas Spt 4 is not essential for growth of budding yeast at $25^{\circ} \mathrm{C}$, deletion of the $s p t 4$ gene prohibits growth at $37^{\circ} \mathrm{C}$ (Malone et al. 1993; Basrai et al. 1996). The available structure-function information for yeast Spt 4 concerns the role of an $\mathrm{N}$-terminal tetracysteine motif that was predicted to comprise a zinc-binding site. Malone et al. (1993) had shown that $S$. cerevisiae $S p t 4$ function in vivo was abolished by single serine substitutions for Cys7, Cys24, or Cys27. Basrai et al. (1996) identified a spontaneous C10Y mutation in yeast Spt4 that also abolished its function in vivo. By contrast, a doublemutation of human Spt4 in which Cys16 and Cys19 were replaced by serine did not have any apparent effect on binding of human Spt4 to human Spt5 or on the reconstitution of a transcriptionally active Spt5-Spt4 (DSIF) complex (Kim et al. 2003). Thus, there are discordant results with respect to the effect of disrupting the putative $\mathrm{Zn}$-binding site of yeast versus human Spt4. Wenzel et al. (2008) verified by optical emission spectrometry that purified human Spt4 contains an equimolar amount of $\mathrm{Zn}^{2+}$. S. pombe Spt4 is notable for its deviation from the tetracysteine motif (Fig. 2B). $S$. pombe Spt4 has three of the conserved cysteines (Cys12, Cys15, and Cys29), but the fourth cysteine is replaced by an aspartate (Asp32).

Previous studies had shown that the ts growth defect of an S. cerevisiae spt4 $\Delta$ strain could be rescued by expressing human or Kluyveromyces lactis Spt4 (Hartzog et al. 1996; Hikkel et al. 1998). Here we constructed an S. cerevisiae spt4d strain and demonstrated rescue of the ts growth defect by expres- sion of S. pombe Spt4 from a CEN plasmid under the control of the yeast TPI1 promoter (Fig. 3A). This allowed us to exploit budding yeast as a surrogate system for structure-function analysis of the 105-amino acid S. pombe Spt4 protein. We introduced Ala-mutations at 18 residues of $S$. pombe Spt4, including the four putative $\mathrm{Zn}^{2+}$ ligands and 14 other amino acids that are conserved in human and S. cerevisiae Spt4 (Fig. 2B). We found that alanine mutations at nine of the conserved residues had no apparent impact on spt4s complementation: Arg10, Gly28, Thr41, Ser42, Gly47, Trp58, Arg61, Trp62, and Gly71 (Fig. 3A). Alanine changes at nine other conserved positions eliminated or reduced $s p t 4 \Delta$ complementation at $37^{\circ} \mathrm{C}$ : Cys 12 , Cys15, Phe24, Cys29, Asn31, Asp32, Phe45, Ser57, and Tyr73 (Fig. 3A).

The S. pombe Spt4-Ala mutants were tested for interaction with $S$. pombe Spt5 in the yeast two-hybrid assay, with dual reporter readouts $\left(\mathrm{His}^{+}\right.$and $\mathrm{lacZ}^{+}$) of a positive interaction between a DNA binding domain (BD) fused to $S$. pombe Spt4 and a transcription activation domain (AD) fused to Spt5-(165-990) or Spt5-(165-400) (Table 1). Whereas all 18 of the Spt4-Ala proteins retained interaction with the larger Spt5-(165-990) protein (Supplemental Fig.
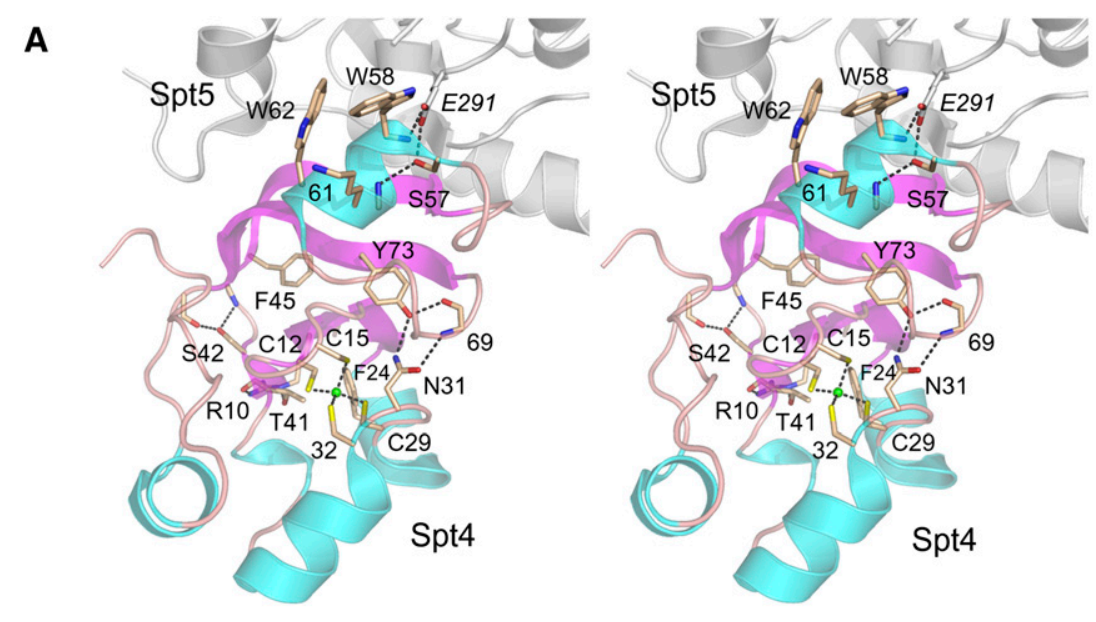

B

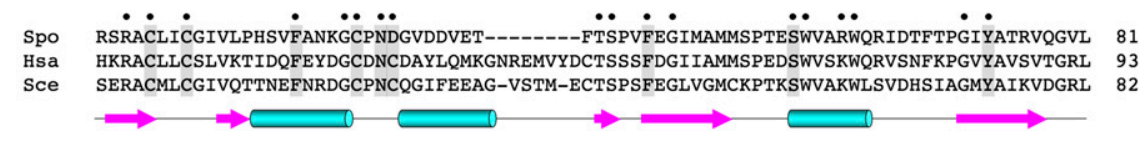

FIGURE 2. Conserved amino acids targeted for alanine scanning in S. pombe Spt4. (A) Location of targeted residues in the Spt4 tertiary structure. The figure (rendered in Pymol from PDB coordinates 2exu) shows a stereo view of a ribbon trace of $S$. cerevisiae Spt4 (with $\beta$ strands colored magenta and $\alpha$-helices colored cyan) in complex with the NGN domains of Spt5 (colored gray). The nonglycine side-chains of Spt4 that were targeted for alanine scanning are shown, numbered according to their position in $S$. pombe $S p t 4$. The $\mathrm{Zn}^{2+}$ ion is depicted as a green sphere. Pertinent atomic contacts are depicted as dashed lines. (B) The amino acid sequence of $S$. pombe (Spo) Spt4 from residues 8-81 is aligned to the sequences of the homologous human (Hsa) and S. cerevisiae (Sce) Spt4 proteins. The secondary structure elements of S. cerevisiae Spt 4 are displayed below the alignment (colored as in panel A). The 18 positions targeted for alanine scanning are indicated by $\bullet$. The nine residues of $S$. pombe Spt 4 that were required for $s p t 4 \Delta$ complementation in S. cerevisiae are highlighted in gray boxes. 


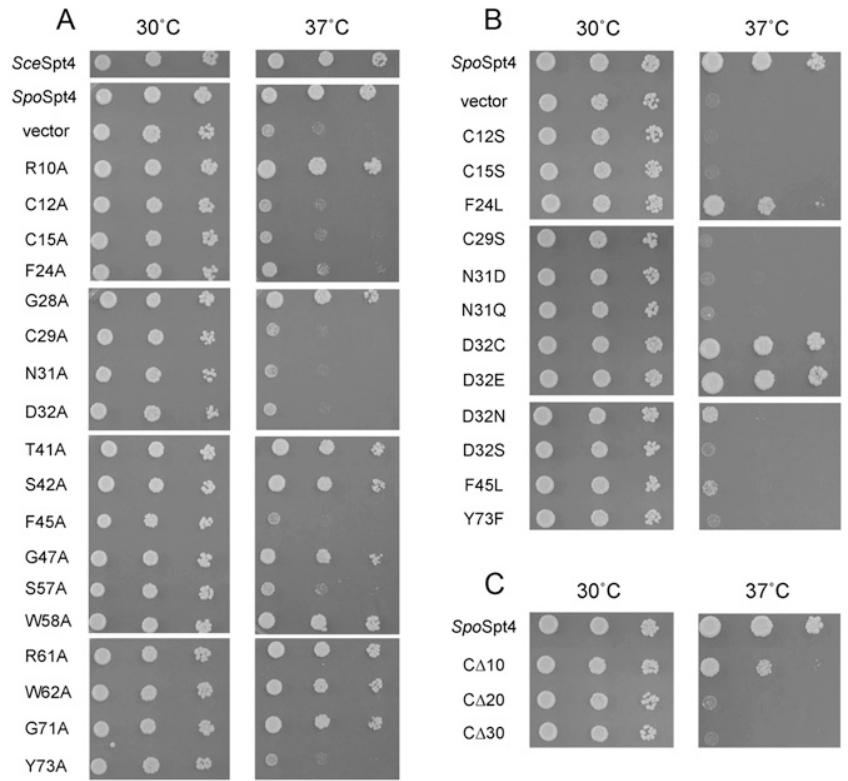

FIGURE 3. Mutational effects on the function of $S$. pombe Spt4 assayed by complementation of $S$. cerevisiae spt4A. CEN TRP1 plasmids expressing wild-type SceSpt4, wild-type SpoSpt4, or mutated

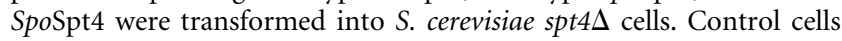
were transformed with the empty CEN TRP1 vector. Growth of the indicated $\operatorname{spt} 4 \Delta$ strains was assessed by spotting serial dilutions to SDTrp agar medium. The plates were photographed after incubation for $3 \mathrm{~d}$ at $30^{\circ} \mathrm{C}$ or $37^{\circ} \mathrm{C}$ as specified. (A) Effects of alanine mutations. (B) Effects of conservative mutations. $(C)$ Effects of C-terminal truncations.

S1), four of the Spt4 mutations (C12A, C15A, C29A, and Y73A) affected Spt4 binding to the smaller Spt5-(165-400) fragment that suffices for binding to wild-type Spt4 and 14 other alanine mutants (Table 1). That the S. pombe Spt4Ala mutants retained activity in one or both two-hybrid assays indicates that the mutant polypeptides are indeed produced in budding yeast.

Structure-activity relationships at the essential residues were gleaned by testing the effects of 13 conservative

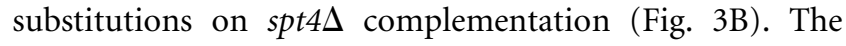
C12S, C15S, and C29S mutations abolished Spt4 activity, suggesting that zinc coordination is essential for the fission yeast protein, as it is in budding yeast Spt4. The instructive finding was that replacing the deviant Asp32 residue of $S$. pombe Spt 4 with either glutamate or cysteine fully restored complementation activity, whereas serine and asparagine did not (Fig. 3B). We surmise that (1) either a cysteine sulfur or a carboxylate oxygen can serve as the fourth metal ligand and (2) there is no tight steric constraint on distance from the main-chain to the metal-binding atom of the side-chain at position 32. Replacing Tyr73 by phenylalanine resulted in loss of function (Fig. 3B), suggesting that the hydroxyl group was essential. Spt4 was active in vivo when Phe24 was substituted by leucine, indicating that the aromatic ring was not essential. By contrast, the aromatic quality of Phe45 was essential, insofar as the F45L mutant failed to complement spt4s (Fig. 3B). The essential function of Asn31 could not be performed by aspartate or glutamine, indicating that the amide group is critical and/or there is a steric constraint on the distance from the main-chain to the amide.

We also tested the effects of serial deletions of 10, 20, 30, 40 , or 50 amino acids from the $\mathrm{C}$ terminus of $S$. pombe Spt4. The C $\Delta 10$ mutant supported slow growth of spt4s cells at $37^{\circ} \mathrm{C}$; the $\mathrm{C} \Delta 20$ and $\mathrm{C} \Delta 30$ versions were inactive (Fig. 3C), notwithstanding that the $\mathrm{C} \Delta 20$ and $\mathrm{C} \Delta 30$ polypeptides retained their interactions with $S$. pombe Spt5(165-990) and Spt5-(165-400) in the two-hybrid assay (Table 1). We infer that the segment of Spt4 from amino acids $86-95 \quad\left({ }^{86}\right.$ VESLRRRGIN $\left.{ }^{95}\right)$ embraces functional group(s) necessary for biological activity at a step other than mere binding to Spt5. Note that Spt5 binding was abolished by the Spt $4 \mathrm{C} \Delta 40$ and $\mathrm{C} \Delta 50$ deletions (Table 1). These results indicate that the Spt4 segment from amino acids $66-75\left({ }^{66}\right.$ DTFTPGIYAT $\left.^{75}\right)$ includes function groups necessary for Spt5 binding, one of which is likely to be Tyr73 (Table 1).

\section{Interpretation of mutational results in light of an Spt4 crystal structure}

A stereo image of the crystal structure of S. cerevisiae Spt4 in complex with a fragment of Spt5 (Guo et al. 2008) is shown in Figure 2A. The conserved amino acid side-chains that were subjected to mutational analysis in the present study are highlighted; for convenience, they are renumbered

TABLE 1. Mutational effects on Spt4 two-hybrid interaction with Spt5

\begin{tabular}{lcc}
\hline BD-SpSpt & AD-SpSpt5-(165-990) & Ad-Spt5-(165-400) \\
\hline WT & + & + \\
R10A & + & + \\
C12A & + & - \\
F24A & + & - \\
G28A & + & + \\
C29A & + & - \\
N31A & + & + \\
D32A & + & + \\
T41A & + & + \\
S42A & + & + \\
F45A & + & + \\
G47A & + & + \\
S57A & + & + \\
W58A & + & + \\
R61A & + & + \\
W62A & + & + \\
G71A & + & + \\
Y73A & + & - \\
C 410 & + & + \\
C 420 & + & + \\
C 430 & + & + \\
C 440 & - & - \\
C 450 & - & - \\
\hline & & + \\
& + & +
\end{tabular}


in the figure according to their positions in the $S$. pombe Spt4 polypeptide. The structure rationalizes the essentiality of several of the side-chains identified presently.

Spt 4 consists of a five-stranded anti-parallel $\beta$ barrel flanked by four $\alpha$ helices (Fig. 2). Spt 4 has a single bound $\mathrm{Zn}^{2+}$ atom coordinated with tetrahedral geometry by the four conserved cysteines (or three cysteines plus Asp32 in $S$. pombe Spt4). The fact that all four zinc ligands are essential for yeast Spt4 function likely reflects the role of the zinc complex in maintaining the proper Spt4 tertiary structure. Our finding that the zinc ligands are not essential for interaction with S. pombe Spt5-(165-990) in the two-hybrid assay is consistent with the fact that the zinc-complex is distant from the Spt4-Spt5 interface revealed by the crystal structure. Yet, the $\mathrm{Zn}^{2+}$-binding cysteines are important for binding to the smaller Spt5-(165-400) domain in the twohybrid assay. It is conceivable that additional stabilizing contacts exist between Spt4 and constituents of Spt5 located distal to the NGN module.

Spt4 and Spt5 pack against each other so that their $\beta$-strands form an extended sheet. In addition, the $\alpha 3$ helix of Spt4 makes polar and hydrophobic contacts with Spt5. Conserved Spt4 residues Trp58, Trp62, and Arg61 (a lysine in $S$. cerevisiae) are disposed on the same face of the helix, and none of them is essential for function per se. However, Ser57 is essential, and it donates a hydrogen bond to a conserved glutamate of Spt5 (Glu291 in S. pombe; Glu338 in S. cerevisiae) (Fig. 2A). This Spt5 glutamate (highlighted in Fig. 1D) makes a bidentate contact with Ser57 O $\gamma$ and the main-chain amide of Trp58 (Fig. 2A). Guo et al. (2008) found that mutation of the $S$. cerevisiae Spt5 Glu388 to lysine (a charge inversion) weakened the Spt5-Spt4 interaction. Essential Spt 4 residues Asn 31 and Tyr73 contribute a network of hydrogen bonds to each other and the Thr69 main-chain amide and carbonyl (Ile70 in S. cerevisiae). Arg10 and Thr41 interact with each other via hydrogen bonding, but neither of these side-chains is required for Spt4 activity. Ser42 makes cross-strand hydrogen bonds that are also apparently not critical for function. The essential Phe24 residue makes van der Waals contacts with several side-chains in the protein interior. The essential Phe45 is surfaceexposed and has no obvious contacts in the crystal structure that explain its contributions to Spt4 activity.

\section{Deletion of Spt4 in S. pombe}

Whereas Spt5 is essential for viability of S. pombe (Pei and Shuman 2002), Spt4 had not been studied genetically in fission yeast. In order to delete the spt $4^{+}$ gene, either an spt4::kanMX cassette or a spt4::natMX cassette was transformed into a diploid strain of $S$. pombe, and chromosomal integrants containing one copy of the wild-type gene and one of either spt4::kanMX or spt4::natMX were selected on medium containing G418 or nourseothricin, respectively. Correct integration was confirmed by diagnostic PCR amplification of genomic DNA from the heterozygotes and by Southern blotting. We then sporulated the heterozygotes, dissected tetrads, and scored for spore viability and the presence of the kanMX or natMX marker. We found that tetrads yielded four viable haploid progeny at $30^{\circ} \mathrm{C}$, two of which were G418-resistant or nourseothricin-resistant. We conclude that Spt4 is not essential for cell growth in S. pombe. However, we found that the $S$. pombe spt4 $\Delta$ mutation elicits a tight $t s$ phenotype, whereby the spt4 $\Delta$ strain grows at $30^{\circ} \mathrm{C}$ but fails to grow at $37^{\circ} \mathrm{C}$ (Fig. 4, spt4::natMX; Supplemental Fig. S2A, spt4::kanMX). Growth at $37^{\circ} \mathrm{C}$ was restored by transformation of $s p t 4 \Delta$ with a plasmid-borne copy of $s p t 4^{+}$driven by the low-strength version of the $n m t 1$ promoter, but not with the empty plasmid vector (Supplemental Fig. S2B). We surveyed the collection of $18 s p t 4^{\text {ala }}$ mutants for their ability to complement the $t s$ growth defect of the spt4 $\Delta$ strain when delivered on a plasmid. The $C 12 A$ and $C 15 A$ strains were sick at $37^{\circ} \mathrm{C}$ (Supplemental Fig. S2B). $R 10 A$ and the 15 other spt $4^{\text {ala }}$ mutants supported apparently normal growth of spt4s cells at $37^{\circ} \mathrm{C}$ (Supplemental Fig. S2B; data not shown).

The discrepancy that seven residues found to be essential

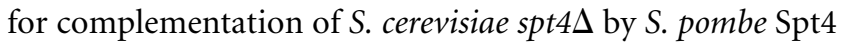
(Phe24, Cys29, Asn31, Asp32, Phe45, Ser57, and Tyr73) were not required to complement Spt4 function in fission yeast could arise because the two genetic assays rely on greatly different gene dosages. In the S. cerevisiae complementation test, the Spt4 proteins are expressed from singlecopy CEN plasmids, but no such single-copy plasmid system
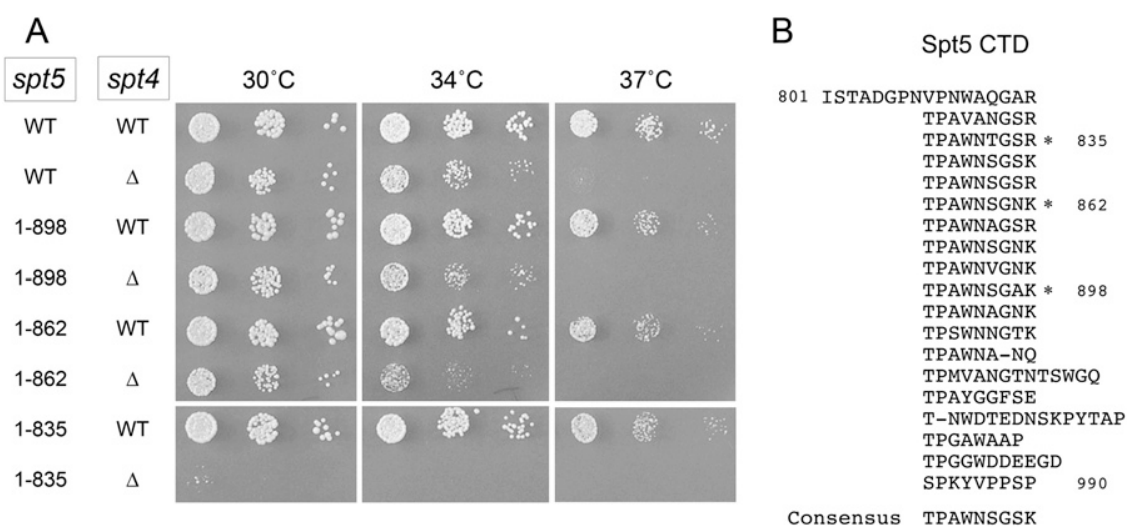

FIGURE 4. Synergy between $s p t 4 \Delta$ and spt5 CTD truncations. (A) Serial dilutions of S. pombe strains bearing the indicated chromosomal spt5 and spt4 alleles were tested for growth on YES agar. The plates were photographed after incubation for $2 \mathrm{~d}\left(\right.$ at $\left.34^{\circ} \mathrm{C}\right)$ or $3 \mathrm{~d}\left(\right.$ at $30^{\circ}$ and $37^{\circ} \mathrm{C}$ ). (B) The Spt5 CTD from amino acids 801-990 is displayed with the nonamer repeats aligned vertically. The consensus sequence TPAWNSGSK is shown below the alignment. The C-termini of the three truncated Spt5 CTDs analyzed in panel $A$ are indicated by asterisks.

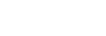

\author{
RNA, Vol. 15, No. 7
}


is available for fission yeast. Instead, we used high-copy vectors that are standard in the field (Forsburg 1993). Therefore, mutations that cause functional deficits at low gene dosage in budding yeast might be missed at high gene dosage in fission yeast, e.g., because increased production of the mutant Spt4 results by mass action in sufficient Spt5Spt 4 complex to support cell growth at $37^{\circ} \mathrm{C}$. We attempted to down-regulate transcription of the plasmid-borne nmt1$s p t 4^{+}$cassette by inclusion of thiamine in the growth medium (Forsburg 1993) and found that the plasmid still complemented growth of the spt $t \Delta$ strain at $37^{\circ} \mathrm{C}$ (data not shown), implying that Spt 4 is indeed produced in functional excess from the high copy plasmid.

An alternative explanation for the discrepancy is that the two genetic complementation assays rely on Spt4 interaction with different Spt5 binding partners. The budding yeast complementation assay is contingent on $S$. pombe Spt4 forming a functional complex with a heterologous $S$. cerevisiae Spt5. The cross-species subunit interaction might be more sensitive to certain Spt4 mutations than the intraspecies interaction. Note that whereas we have shown here that wild-type $S$. cerevisiae and S. pombe Spt4 proteins are functionally interchangeable in budding yeast, this is not the case for the Spt5 proteins. Specifically, we constructed a $S$. cerevisiae spt5 $\mathrm{p}(C E N$ URA3 SPT5) strain suitable for assay of essential Spt 5 functions by the plasmid shuffle method. We found that a plasmid-borne S. cerevisiae SPT5 gene complemented the null mutant. By contrast, a plasmid expressing full-length $S$. pombe Spt5 under the control of a constitutive $S$. cerevisiae promoter did not complement in this assay, even when cotransformed with a plasmid expressing S. pombe Spt4 (data not shown).

\section{Genetic interactions between Spt4 and the Spt5 CTD}

S. pombe Spt5 is distinguished by the orderly primary structure of its CTD, which extends from amino acids 801990 and consists of a tandem array of nonapeptide repeats of the consensus sequence TPAWNSGK (Fig. 4B). The unmodified Spt5 CTD binds avidly to the triphosphatase and guanylyltransferase components of the $S$. pombe capping apparatus (Pei and Shuman 2002). The Spt5 CTD is also a substrate for threonine phosphorylation by $S$. pombe Cdk9 (Pei and Shuman 2003). Here we interrogated genetically the role of the Spt5 CTD nonamer array, by constructing mutant strains of $S$. pombe in which spt5 alleles encoding incrementally truncated Spt 5 proteins were integrated at the chromosomal spt5 locus in lieu of the wild-type gene. The Spt5-(1-898), Spt5-(1-862), and Spt5(1-835) proteins contain rump CTDs with ten, six, and three nonamer repeats, respectively (Fig. 4B). Full-length Spt5 and each of the three Spt5-CTD $\Delta$ proteins were expressed in $S$. pombe at comparable steady-state levels, as gauged by Western blotting of whole-cell lysates with purified polyclonal Spt5 antibody (Supplemental Fig. S3).
The immunoreactive Spt5 proteins displayed the expected incremental changes in electrophoretic mobility (Supplemental Fig. S3). We found that haploid S. pombe strains bearing any of the three truncated spt5 alleles grew as well as the $\operatorname{spt5^{+}}$ strain at $30^{\circ} \mathrm{C}, 34^{\circ} \mathrm{C}$, and $37^{\circ} \mathrm{C}$, as gauged by colony size (Fig. 4A). We conclude that three nonamer repeats (containing two Cdk9 Thr-Pro phosphorylation sites) suffice for viability under laboratory growth conditions. Complete deletion of all nonamer repeats causes an overt growth defect that will be described in detail elsewhere (S Schneider and B Schwer, in prep.).

The instructive finding here was that combining the serial

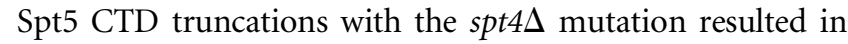
slowed growth at temperatures normally permissive for the $s p t 4 \Delta$ strain (Fig. 4A, spt $5^{1-862} s p t 4 \Delta$ ). This synthetic enhancement culminated in the lethality of the $s p t 5^{1-835} s p t 4 \Delta$ double mutant at $34^{\circ} \mathrm{C}$ and a strong synthetic sick phenotype at $30^{\circ} \mathrm{C}$ (Fig. $4 \mathrm{~A}$ ). We surmise that a longer Spt5 CTD becomes essential when Spt 4 function is ablated. The implication is that interaction of Spt 4 with the central domain of Spt5 overlaps functionally with the Spt5 CTD.

\section{Concluding remarks}

The present study extends our knowledge of the fission yeast core Spt5-Spt4 complex, which we characterize as a heterodimer. Fission yeast Spt5 has an exceptionally regular CTD nonapeptide array that mediates Spt5 interactions with mRNA capping enzymes. Here we showed that as few as three nonamer repeats sufficed for cell growth, but only when Spt4 was present. A genetic analysis of Spt4 in $S$. pombe revealed it to be inessential for growth at $25^{\circ} \mathrm{C}-$ $30^{\circ} \mathrm{C}$, but critical at $37^{\circ} \mathrm{C}$. These results echo the temperature-sensitive spt $4 \Delta$ growth phenotype in budding yeast, where S. cerevisiae and S. pombe Spt4 are functionally interchangeable. The $S$. cerevisiae complementation assay provided a sensitive readout of mutational effects on $S$. pombe Spt4 function in vivo. The targets for the Spt4 alanine scan were chosen based on conservation among eukaryal and archaeal Spt4 homologs. After the mutational study was completed, the coordinates of the crystal structure of S. cerevisiae Spt4 bound to a fragment of Spt5 were deposited (RCSB Protein Databank ID code 2exu). The structure, now published (Guo et al. 2008), provided an invaluable guide to interpret the mutational data we obtained for S. pombe Spt4. The genetic tools described here can assist further dissection of the postulated transcription elongation checkpoint in fission yeast.

\section{MATERIALS AND METHODS}

\section{Recombinant S. pombe Spt5-Spt4 complex}

The fission yeast Spt4 and Spt5-(165-800) ORFs were inserted into the pETDuet expression vector (Novagen) so that the Spt4 ORF 
was fused in-frame to a leader sequence encoding a His-tag. The dual-expression plasmid was transformed into Escherichia coli BL21(DE3). Expression of the $S$. pombe proteins was induced by IPTG, and the His-tagged Spt4 and associated Spt5-(165-800) were purified from a soluble bacterial lysate by $\mathrm{Ni}$-agarose chromatography, following protocols developed previously for other recombinant His-tagged S. pombe proteins (Pei et al. 2001). The affinity-purified HisSpt4/Spt5-(165-800) complex was mixed with marker protein catalase, BSA and vtopo. The mixture was applied to a $4.8-\mathrm{mL} 15 \%-30 \%$ glycerol gradient containing 50 $\mathrm{mM}$ Tris- $\mathrm{HCl}$ ( $\mathrm{pH} 8.0), 125 \mathrm{mM} \mathrm{NaCl}, 1 \mathrm{mM}$ EDTA, and $0.05 \%$ Triton-X100. The gradient was centrifuged in an SW50 rotor at $50,000 \mathrm{rpm}$ for $18 \mathrm{~h}$ at $4^{\circ} \mathrm{C}$. Fractions $(0.2 \mathrm{~mL})$ were collected from the bottom of the tube. An aliquot of glycerol gradient fraction 15 containing the putative HisSpt4/Spt5-(165-800) heterodimer was subjected to a second round of Ni-affinity purification as described (Pei and Shuman 2002).

\section{Media and genetic manipulations}

We followed standard protocols for yeast growth, transformations, mating, sporulation, and tetrad dissection, as described by Guthrie and Fink (1991) and Schiestl and Gietz (1989) for S. cerevisiae and on the Forsburg laboratory Pombe Technology website (www-rcf.usc.edu/ forsburg/index.html) for S. pombe.

\section{Genetic assay of Spt4 function in S. cerevisiae}

The S. cerevisiae SPT4 open reading frame (ORF) was replaced in a diploid W303 strain with a spt4::kanMX gene disruption cassette (Güldener et al. 1996). Integrants were selected on YPD agar plates containing geneticin $(150 \mathrm{mg} / \mathrm{L}$, Invitrogen). Correct gene targeting was confirmed by diagnostic Southern blotting of restriction endonuclease-digested genomic DNA. The SPT4 spt4A diploid was sporulated, and tetrads were dissected. We thereby obtained a haploid spt4s strain (Mata spt4::kanMX ura3 ade2 trp1 his3 leu2 can1) that grew well at $30^{\circ} \mathrm{C}$ but failed to form colonies on YPD agar at $37^{\circ} \mathrm{C}$. A complementing plasmid, p358SceSpt4, was generated by inserting a genomic NheI-EcoRI fragment $(2.3 \mathrm{~kb})$ containing the wild-type S. cerevisiae SPT4 gene into pSE358 (CEN TRP1). For expression of $S$. pombe Spt4 in budding yeast, a DNA fragment spanning the 318-bp spt4 $4^{+}$ORF was amplified from chromosomal DNA by PCR with primers designed to introduce an NdeI site at the ATG codon and a BamHI site downstream of the stop codon. The DNA fragment was restricted and inserted into pYN132 (TRP1 CEN), a shuttle vector (modified from pYX132, Novagen) in which expression of $S$. pombe Spt4 is under the transcriptional control of the constitutive TPI1 promoter. Alanine mutations were introduced into the wild-type $s p t 4^{+}$gene by two-stage overlap extension PCR with mutagenic primers and pYN132-spt4 as the template. The mutated alleles were restricted and inserted into pYN132. The inserts of all plasmids were sequenced to verify that no undesired coding changes were introduced during amplification and cloning. The CEN TRP1 plasmids expressing wild-type SceSpt4, wildtype SpoSpt4, or mutated SpoSpt4 were transformed into $S$. cerevisiae spt $4 \Delta$ cells. Growth of $\operatorname{Trp}^{+}$transformants was assessed by spotting $3 \mu \mathrm{L}$ aliquots of serial 10 -fold dilutions of yeast cultures (grown to mid-log phase in synthetic dropout [SD] broth lacking tryptophan at $30^{\circ} \mathrm{C}$ and adjusted to $A_{600}$ of 0.1 ) to SD-Trp agar medium and then incubating the plates at $30^{\circ} \mathrm{C}$ and $37^{\circ} \mathrm{C}$.

\section{Two-hybrid assay of Spt4-Spt5 interaction}

The two-hybrid assays were performed as described previously (Pei et al. 2001; Pei and Shuman 2002). Briefly, aliquots of S. cerevisiae Y190 cells (Clontech Laboratories) harboring plasmids for expression of Gal4(AD)-Spt5-(165-990) or Gal4(AD)-Spt5-(165-400) plus wild-type or mutated Gal4(BD)-Spt4 proteins were tested for histidine prototrophy by spotting to $\mathrm{SD}(-\mathrm{Trp}$, -Leu, -His) agar medium containing $25 \mathrm{mM} 3$-amino-1,2,4-triazole (Sigma). Growth was scored after $4 \mathrm{~d}$ of incubation at $30^{\circ} \mathrm{C}$. Expression of the lac $Z$ reporter was scored by blue color in the $\beta$-galactosidase colony-lift filter assay.

\section{Deletion of spt4 in S. pombe}

The entire $s p t 4^{+}$ORF was replaced in a diploid strain with either the kanMX or natMX gene conferring antibiotic resistance. To construct the disruption cassettes, DNA segments spanning 490 bp upstream and $410 \mathrm{bp}$ downstream of the spt4 ORF were amplified by PCR from chromosomal DNA and inserted into modified pTOPO vectors to flank the kanMX (Güldener et al. 1996) or the natMX (Goldstein and McCusker 1999) cassette. DNA fragments with the spt4::kanMX $(2.4 \mathrm{~kb})$ and spt4::natMX $(2.1 \mathrm{~kb})$ disruption cassettes were transformed into a $S$. pombe diploid. Transformants were selected on YES (yeast extracts with supplements) agar medium containing geneticin (150 mg/L, Invitrogen) or nourseothricin (100 mg/L clonNAT, Werner Bioagents). Correct gene targeting was confirmed by diagnostic colony PCR and Southern blotting. The heterozygous diploids were then sporulated, and tetrads were dissected to obtain spt4 $\Delta$ haploids used for complementation assays.

For expression of Spt4 in fission yeast, DNA fragments encoding wild-type or mutant Spt4 proteins were excised from the respective pYN132-spt4 plasmids and inserted into pREP81x, wherein Spt4 is expressed under the transcriptional control of a weakened nmt1 promoter (Forsburg 1993). Leu ${ }^{+}$transformants were selected and the growth of the various pREP81x-spt4 strains was compared to $s p t 4 \Delta$ cells that had been transformed with the empty pREP81x plasmid. Aliquots $(3 \mu \mathrm{L})$ of serial 10 -fold dilutions of $S$. pombe cultures (grown to mid-log phase in SD broth lacking leucine at $30^{\circ} \mathrm{C}$ and adjusted to $A_{600}$ of 0.1 ) were spotted on to agar medium and the plates were incubated at $30^{\circ}$ and $37^{\circ} \mathrm{C}$.

\section{Truncations of the Spt5 CTD array in S. pombe}

We constructed a pUC19-based plasmid harboring the $u r a 4^{+}$gene upstream of a 534-bp fragment of $S$. pombe genomic DNA located immediately $3^{\prime}$ of the stop codon of the $s p t 5^{+}$gene. We then inserted upstream of $\mathrm{ura}^{+}$a series of $s p t 5$ gene fragments extending from an NdeI site (at nucleotide +1736 within the spt5 ORF) to either the stop codon at position +2971 (the natural stop codon) or newly created stop codons at positions $+2695,+2587$, or +2506 that result in truncations of the Spt5 CTD. Linearized spt5 $5^{C T D+/ \Delta}-u r a 4^{+}-$ $s p t 5^{3^{\prime}}$ cassettes were transformed into haploid $S$. pombe cells. $\mathrm{Ura}^{+}$ transformants were selected and targeted insertions were verified by diagnostic Southern blotting.

\section{Anti-Spt5 antibodies}

Polyclonal antibodies were raised in rabbits immunized with recombinant His $_{10}$ Smt3-Spt5-(1-990) at the Pocono Rabbit Farm 
and Laboratory (Canadensis, PA). Anti-Spt5 antibodies were affinity purified from whole serum by affinity-chromatography by adsorption to $\mathrm{His}_{10} \mathrm{Smt3}-\mathrm{Spt5}$-(1-990) coupled to Affigel-10 (Bio-Rad).

\section{Western blot analysis}

The haploid $\left(\mathrm{h}^{-}\right) \quad s p t 5^{C T D+/ \Delta}-u r a 4^{+}-s p t 5^{3^{\prime}}$ strains spt5-(1-

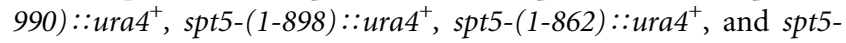
(1-835) ::ura $4^{+}$were grown in YES broth at $30^{\circ} \mathrm{C}$. Aliquots of cells from exponentially growing cultures (equivalent to $6 A_{600}$ units) were harvested by centrifugation. The cell pellets were suspended in $200 \mu \mathrm{L}$ SDS-sample buffer (2\% SDS, $50 \mathrm{mM}$ Tris- $\mathrm{HCl}$ at $\mathrm{pH}$ 6.8, $100 \mathrm{mM}$ DTT, $0.1 \%$ bromphenol blue, $10 \%$ glycerol), and acid-washed glass beads $(\sim 100 \mu \mathrm{L})$ were added. Cells were disrupted by five 30 -sec cycles of vigorous vortexing. The samples were heated for $5 \mathrm{~min}$ at $100^{\circ} \mathrm{C}$ and centrifuged to remove macroaggregates and glass beads. Aliquots $(25 \mu \mathrm{L})$ of the wholecell lysates were analyzed by $6 \%$ SDS-PAGE and stained with Coomassie brilliant blue to verify that similar amounts of total protein were present in each of the lysates. For Western blot analysis, aliquots $(2 \mu \mathrm{L})$ of the lysate were resolved by $6 \%$ SDSPAGE, and the polypeptides were transferred to a PVDF membrane (NEN Research Products) with a semi-dry transfer apparatus (semi-phor, Hoefer Scientific Instruments). The membrane was probed with affinity purified anti-Spt5 antibodies, and the immune complexes were visualized with horseradish peroxidaseconjugated anti-rabbit immunoglobulin (Cell Signaling Technology) using an enhanced chemiluminescence system (ECL, Amersham).

\section{Mutational synergy between $s p t 4 \Delta$ and $s p t 5^{\mathrm{CTD} \Delta}$}

Haploid spt4s::natMX $\left(\mathrm{h}^{+}\right)$cells were mated to each of the following haploid $\left(\mathrm{h}^{-}\right) s p t 5^{C T D+/ \Delta}-u r a 4^{+}-s p t 5^{3^{\prime}}$ strains: spt5-(1990)::ura4 $4^{+}$, spt5-(1-898)::ura4 ${ }^{+}$, spt5-(1-862)::ura4 ${ }^{+}$, and spt5(1-835)::ura $4^{+}$. Diploids were selected on synthetic medium lacking uracil and containing nourseothricin. The diploids were sporulated and tetrads dissected. The genotypes of haploid progeny were confirmed by diagnostic colony PCR. Growth of the $s p t 5^{\mathrm{CTD}+/ \Delta}$ and the $s p t 5^{\mathrm{CTD}+/ \Delta} \operatorname{spt} t \Delta$ strains was assessed by spotting $3 \mu \mathrm{L}$ aliquots of serial 10-fold dilutions of logarithmic cultures grown at $30^{\circ} \mathrm{C}$ onto YES agar medium and then incubating the plates at $30^{\circ} \mathrm{C}, 34^{\circ} \mathrm{C}$, or $37^{\circ} \mathrm{C}$.

\section{SUPPLEMENTAL MATERIAL}

Supplemental material can be found at http://www.rnajournal.org.

\section{ACKNOWLEDGMENTS}

This work was supported by National Institutes of Health grant GM52470 (B.S. and S.S.). S.S. is an American Cancer Society Research Professor.

Received January 22, 2009; accepted April 2, 2009.

\section{REFERENCES}

Andrulis ED, Guzman E, Döring P, Werner J, Lis JT. 2000. Highresolution localization of Drosophila Spt5 Spt6 at heat shock genes in vivo: Roles in promoter proximal pausing and transcription elongation. Genes \& Dev 14: 2635-2649.

Basrai MA, Kingsbury J, Koshland D, Spencer F, Hieter P. 1996. Faithful chromosome transmission requires $\mathrm{Spt} 4 \mathrm{p}$, a putative regulator of chromatin structure in Saccharomyces cerevisiae. Mol Cell Biol 16: 2838-2847.

Chiu YL, Ho CK, Saha N, Schwer B, Shuman S, Rana TM. 2002. Tat stimulates cotranscriptional capping of HIV mRNA. Mol Cell 10: 585-597.

Cho E, Takagi T, Moore CR, Buratowski S. 1997. mRNA capping enzyme is recruited to the transcription complex by phosphorylation of the RNA polymerase II carboxyl-terminal domain. Genes \& Dev 11: 3319-3326.

Fabrega C, Shen V, Shuman S, Lima CD. 2003. Structure of an mRNA capping enzyme bound to the phosphorylated carboxyl-terminal domain of RNA polymerase II. Mol Cell 11: 1549-1561.

Forsburg SL. 1993. Comparison of Schizosaccharomyces pombe expression systems. Nucleic Acids Res 21: 2955-2956.

Gao L, Gross DS. 2008. Sir2 silences gene transcription by targeting the transition between RNA polymerase II initiation and elongation. Mol Cell Biol 28: 3979-3994.

Goldstein AL, McCusker JH. 1999. Three new dominant drug resistance cassettes for gene disruption in Saccharomyces cerevisiae. Yeast 15: 1541-1553.

Guiguen A, Soutourine J, Dewez M, Tafforeau L, Dieu M, Raes M, Vandenhaute J, Werner M, Hermand D. 2007. Recruitment of P-TEFb (Cdk9-Pch1) to chromatin by the cap-methyl transferase Pcm1 in fission yeast. EMBO J 26: 1552-1559.

Güldener U, Heck S, Fielder T, Beinhauer J, Hegemann JH. 1996. A new efficient gene disruption cassette for repeated use in budding yeast. Nucleic Acids Res 24: 2519-2524.

Guo S, Yamaguchi Y, Schilbach S, Wada T, Lee J, Goddard A, French D, Handa H, Rosenthal A. 2000. A regulator of transcription elongation controls vertebrate neuronal development. Nature 408: 366-369.

Guo M, Xu F, Yamada J, Egelhofer T, Gao Y, Kartzog GA, Teng M, Niu L. 2008. Core structure of the yeast Spt4-Spt5 complex: A conserved module for regulation of transcription elongation. Structure 16: 1649-1658.

Guthrie C, Fink G. 1991. Guide to yeast genetics and molecular biology. Academic Press, San Diego, CA.

Hartzog GA, Basrai MA, Ricupero-Hovasse SL, Hieter P, Winston F. 1996. Identification and analysis of a functional human homolog of the SPT4 gene of Saccharomyces cerevisiae. Mol Cell Biol 16: 2848-2856.

Hartzog GA, Wada T, Handa H, Winston F. 1998. Evidence that Spt4, Spt5, and Spt6 control transcription elongation by RNA polymerase II in Saccharomyces cerevisiae. Genes \& Dev 12: 357-369.

Hikkel I, Gbelska Y, Subik J. 1998. Identification and functional analysis of a Kluyveromyces lactis homologue of the SPT4 gene of Saccharomyces cerevisiae. Curr Genet 34: 375-378.

Ho CK, Shuman S. 1999. Distinct roles for CTD Ser2 and Ser5 phosphorylation in the recruitment and allosteric activation of mammalian capping enzyme. Mol Cell 3: 405-411.

Ivanov D, Kwak YT, Guo J, Gaynor RB. 2000. Domains in the Spt5 protein that modulate its transcriptional regulatory properties. Mol Cell Biol 20: 2970-2983.

Jennings BH, Shah S, Yamaguchi Y, Seki M, Phillips RG, Handa H, Ish-Horowicz D. 2004. Locus-specific requirements for Spt5 in transcriptional activation and repression in Drosophila. Curr Biol 14: $1680-1684$.

Kim D, Inukai N, Yamada T, Furuya A, Sata H, Yamaguchi Y, Wada T, Handa H. 2003. Structure-function analysis of human Spt4: Evidence that hSpt4 and hSp5 exert their roles in transcriptional elongation as parts of the DSIF complex. Genes Cells 8: $371-$ 378.

Lehman K, Schwer B, Ho CK, Rouzankina I, Shuman S. 1999. A conserved domain of yeast RNA triphosphatase flanking the catalytic core regulates self-association and interaction with the 
guanylyltransferase component of the mRNA capping apparatus. J Biol Chem 274: 22668-22678.

Lindstrom DL, Squazzo SL, Mister N, Burckin TA, Wachter KC, Emigh CA, McCleery JA, Yates JR, Hartzog GA. 2003. Dual roles for Spt5 in pre-mRNA processing and transcription elongation revealed by identification of Spt5-associated proteins. Mol Cell Biol 23: $1368-1378$.

Malone EA, Fassler JS, Winston F. 1993. Molecular and genetic characterization of SPT4, a gene important for transcription initiation in Saccharomyces cerevisiae. Mol Gen Genet 237: 449-459.

Mandal SS, Chu C, Wada T, Handa H, Shatkin AJ, Reinberg D. 2004. Functional interaction of RNA-capping enzyme with factors that positively and negatively regulate promoter escape by RNA polymerase II. Proc Natl Acad Sci 101: 7572-7577.

Mason PB, Struhl K. 2005. Distinction and relationship between elongation rate and processivity of RNA polymerase II in vivo. Mol Cell 17: 831-840.

McCracken S, Fong N, Rosonina E, Yankulov K, Brothers G, Siderovski D, Hessel A, Foster S, Shuman S, Bentley DL. 1997. 5' Capping enzymes are targeted to pre-mRNA by binding to the phosphorylated C-terminal domain of RNA polymerase II. Genes \& Dev 11: 3306-3318.

Pei Y, Shuman S. 2002. Interactions between fission yeast mRNA capping enzymes and elongation factor Spt5. J Biol Chem 277: 19639-19648.

Pei Y, Shuman S. 2003. Characterization of the Schizosaccharomyces pombe Cdk9/Pch1 protein kinase: Spt5 phosphorylation, autophosphorylation and mutational analysis. J Biol Chem 278: 4334643356.

Pei Y, Hausmann S, Ho CK, Schwer B, Shuman S. 2001. The length, phosphorylation state, and primary structure of the RNA polymerase II carboxyl-terminal domain dictate interactions with mRNA capping enzymes. J Biol Chem 276: 28075-28082.

Pei Y, Schwer B, Shuman S. 2003. Interactions between fission yeast Cdk9, its cyclin partner Pch1, and mRNA capping enzyme Pct1 suggest an elongation checkpoint for mRNA quality control. J Biol Chem 278: 7180-7188.

Pei Y, Du H, Singer J, St. Amour C, Granitto S, Shuman S, Fisher RP. 2006. Cdk9 of fission yeast is activated by the CDK-activating kinase Csk1, overlaps functionally with the TFIIH-associated kinase Mcs6, and associates with the cap methyltransferase Pcml in vivo. $\mathrm{Mol}$ Cell Biol 26: 777-788.

Ponting CP. 2002. Novel domains and orthologues of eukaryotic transcription elongation factors. Nucleic Acids Res 30: 3643-3652.

Qui H, Hu C, Wong CM, Hinnebusch AG. 2006. The Spt4p subunit of yeast DSIF stimulates association of the Pafl complex with elongating RNA polymerase II. Mol Cell Biol 26: 3135-3148.

Renner DB, Yamaguchi Y, Wada T, Handa H, Price DH. 2001. A highly purified RNA polymerase II elongation control system. J Biol Chem 276: 42601-42609.
Rondón AG, García-Rubio M, González-Barrera S, Aguilera A. 2003. Molecular evidence for a positive role of Spt4 in transcription elongation. EMBO J 22: 612-620.

Schiestl RH, Gietz RD. 1989. High efficiency transformation of intact yeast cells using single stranded nucleic acids as a carrier. Curr Genet 16: 339-346.

Schneider DA, French SL, Osheim YN, Bailey AO, Lu V, Dodd J, Yates JR, Beyer AL, Nomura M. 2006. RNA polymerase II elongation factors Spt4p and Spt5p play roles in transcription elongation by RNA polymerase I and rRNA processing. Proc Natl Acad Sci 103: 12707-12712.

Shim EY, Walker AK, Shi Y, Blackwell KT. 2002. CDK-9/cyclin T (P$\mathrm{TEFb})$ is required in two postinitiation pathways for transcription in the C. elegans embryo. Genes \& Dev 16: 2135-2146.

Sims RJ, Belotserkovskaya R, Reinberg D. 2004. Elongation by RNA polymerase II: The short and long of it. Genes \& Dev 18: 24372468.

Swanson MS, Malone EA, Winston F. 1991. SPT5, and essential gene important for normal transcription in Saccharomyces cerevisiae, encodes an acidic nuclear protein with a carboxy-terminal repeat. Mol Cell Biol 11: 3009-3019.

Wada T, Takagi T, Yamaguchi Y, Ferdous A, Imai T, Hirose S, Sugimoto S, Yano K, Hartzog GA, Winston F, et al. 1998a. DSIF, a novel transcription elongation factor that regulates RNA polymerase II processivity, is composed of human Spt4 and Spt5 homologs. Genes \& Dev 12: 343-356.

Wada T, Takagi T, Yamaguchi Y, Watanabe D, Handa H. 1998b. Evidence that $\mathrm{P}-\mathrm{TEFb}$ alleviates the negative effect of DSIF on RNA polymerase II-dependent transcription in vitro. EMBO J 17: 7395-7403.

Wen Y, Shatkin AJ. 1999. Transcription elongation factor hSpt5 stimulates mRNA capping. Genes \& Dev 13: 1774-1779.

Wenzel S, Schweimer K, Rösch P, Wöhlri BM. 2008. The small hSpt4 subnit of the human transcription elongation factor DSIF is a Znfinger protein with $\alpha / \beta$-type topology. Biochem Biophys Res Commun 370: 414-418.

Wu CH, Yamaguchi Y, Benjamin LR, Horvat-Gordon M, Washinky J, Enerly E, Larsson J, Lambertsson A, Handa H, Gilmour D. 2003. NELF and DSIF cause promoter proximal pausing on the $h s p 70$ promoter in Drosophila. Genes \& Dev 17: 1402-1414.

Yamada T, Yamaguchi Y, Inukai N, Okamoto S, Mura T, Handa H. 2006. P-TEFb-mediated phosphorylation of hSpt5 C-terminal repeats is critical for processive transcription elongation. Mol Cell 21: 227-237.

Yamaguchi Y, Wada T, Watanabe D, Takagi T, Hasegawa J, Handa H. 1999. Structure and function of the human transcription elongation factor DSIF. J Biol Chem 274: 8085-8092.

Yue Z, Maldonado E, Pillutla R, Cho H, Reinberg D, Shatkin AJ. 1997. Mammalian capping enzyme complements mutant $S$. cerevisiae lacking mRNA guanylyltransferase and selectively binds the elongating form of RNA polymerase II. Proc Natl Acad Sci 94: 12898-12903. 

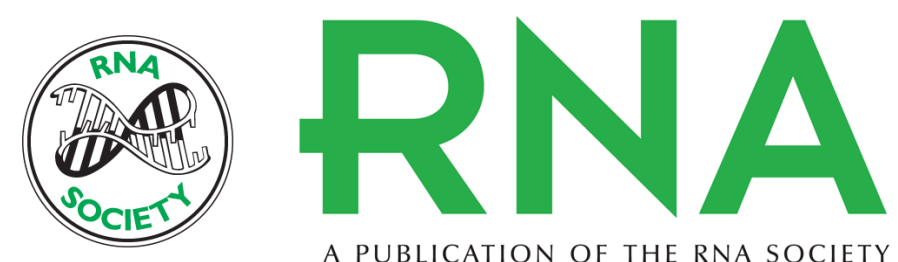

A PUBLICATION OF THE RNA SOCIETY

\section{Characterization of the Schizosaccharomyces pombe Spt5-Spt4 complex}

Beate Schwer, Susanne Schneider, Yi Pei, et al.

RNA 2009 15: 1241-1250 originally published online May 21, 2009

Access the most recent version at doi:10.1261/rna.1572709

\section{Supplemental http://rnajournal.cshlp.org/content/suppl/2009/05/21/rna.1572709.DC1 \\ Material}

References This article cites 47 articles, 29 of which can be accessed free at: http://rnajournal.cshlp.org/content/15/7/1241.full.html\#ref-list-1

\section{License}

Email Alerting Receive free email alerts when new articles cite this article - sign up in the box at the Service top right corner of the article or click here. 